\title{
Infección por Bartonella henselae con compromiso de sistema nervioso central, asociado a un papiloma del plexo coroídeo
}

\author{
Natalia Barnafi, Natalia Conca, Cecilia von Borries, Isabel Fuentes, Francisca Montoya y Elisa Alcalde
}

\section{Central nervous system infection by Bartonella henselae associated with a choroid plexus papilloma}

Bartonella henselae infection is a prevalent illness in Chile. It presents generally as a cat scratch disease or as a prolonged fever syndrome. There are atypical manifestations, which include central nervous system, bone, cardiac and hepato-esplenic compromised. We present an adolescent case with a history of fever, vomiting and ataxia, whose diagnosis was a central nervous system infection by Bartonella henselae associated with a choroid plexus papilloma. This case corresponds to an unusual presentation, with a challenging diagnosis. It is controversial whether to treat this patient, which antimicrobial is the right choice and how long the treatment should be.

Key words: Bartonella infection, central nervous system, granuloma, papilloma

Palabras clave: Infección por Bartonella henselae, sistema nervioso central, granuloma, papiloma.

\section{Introducción}

$\mathrm{E}$ 1 género Bartonella comprende bacilos gramnegativos intracelulares, facultativos, de crecimiento fastidioso. Pertenece taxonómicamente al phylum Proteobacteria $^{1,2}$. Está distribuido mundialmente y es responsable de una variada sintomatología, tanto en hospederos inmunocompetentes como inmunocomprometidos ${ }^{1}$. Existen más de 20 especies descritas y al menos seis son responsables de causar enfermedad en humanos; de ellas, la más difundida es Bartonella henselae ${ }^{1,3,4}$. Existen manifestaciones típicas de la enfermedad como la enfermedad por arañazo de gato, y otras atípicas dentro de las cuales se encuentra el compromiso óseo, hepatoesplénico, del sistema nervioso central (SNC), entre otras. Comunicamos el caso de una adolescente que se manifestó con compromiso del SNC, una presentación inusual.

\section{Caso clínico}

Adolescente de 13 años proveniente de la ciudad de Arica, correspondiente al área costera del norte de Chile, sin antecedentes mórbidos relevantes. Residía en el área urbana con sus padres y hermana. Consultó en el Servicio de Urgencia por una historia de fiebre intermitente de ocho días, con temperatura máxima de $39^{\circ} \mathrm{C}$ axilar, que cedía parcialmente con la administración de paracetamol, asociado a cefalea holocránea. En la anamnesis dirigida refirió contacto directo con gatos mayores de 1 año de edad. Al examen físico se encontraba en buenas condiciones generales, con adenopatías occipitales pequeñas de
$1 \mathrm{~cm}$, blandas, no dolorosas, sin evidencia de rasguños de gato.

Entre los exámenes de laboratorio de ingreso destacaba una proteína $\mathrm{C}$ reactiva (PCR) de $28 \mathrm{mg} / \mathrm{L}$ y una VHS de $75 \mathrm{~mm} / \mathrm{h}$. Dado los antecedentes epidemiológicos y la presentación clínica, se diagnosticó una probable enfermedad por arañazo de gato y se indicó tratamiento con azitromicina por 7 días. A la semana siguiente persistió con fiebre, asociándose diplopia y ataxia, por lo que se decidió internar. Se realizó una punción lumbar (PL), que mostró una pleocitosis de 430 céls $/ \mathrm{mm}^{3}$ con $47 \%$ de mononucleares. El cultivo bacteriológico del LCR resultó negativo.

El estudio imagenológico se inició con una tomografía computada (TC) de encéfalo que demostró un proceso expansivo del cuarto ventrículo, con algunas calcificaciones puntiformes. Se complementó el estudio con una resonancia magnética (RM) que demostró un proceso expansivo centrado en el cuarto ventrículo, hiperintenso en secuencias ponderadas en T2 (T2-WI), isointenso en secuencias ponderadas en T1 (T1-WI), sin restricción de la difusión ni artefacto de susceptibilidad. La lesión presentó un realce homogéneo en secuencias contrastadas (T1Gd-WI). Adicionalmente se observó un aumento del tamaño hipofisiario asociado a incremento de tamaño y de realce del tallo hipofisiario, sugerente de una hipofisitis. Además, presentaba múltiples lesiones nodulares cervicales posteriores, lateralizadas a izquierda, con aspecto de adenopatías (Figura 1).

Se realizó la exéresis tumoral. Evolucionó afebril, con náuseas y vómitos diarios, sin cefalea, con dificultad en la marcha, descartándose un origen endocrinológico,
Facultad de Medicina, Universidad de Chile. Programa de formación de Infectología Pediátrica (NB). Departamento de Pediatría y Cirugía Infantil Campus Oriente (NC).

Programa de formación de especialistas en Pediatría (CVB, IF). Instituto de Neurocirugía. Neurología Adulto y Neurorradiología (FM). Hospital Luis Calvo Mackenna. Anatomía Patológica (EA)

Las autoras declaran no tener conflictos de interés. No se requirió fuente de financiamiento.

Recibido: 31 de octubre de 2016 Aceptado: 18 de mayo de 2017

Correspondencia a: Natalia Barnafi nataliabarnafi@gmail.com 
otorrinolaringológico y gastroenterológico de su sintomatología. Cursó con un síndrome ansioso que fue tratado con fluoxetina.

El estudio histológico de la biopsia tumoral evidenció hallazgos compatibles con un tumor tipo papiloma del plexo coroídeo grado I de la Organización Mundial de la Salud (OMS) y un proceso inflamatorio granulomatoso crónico que rodeaba el tumor, con bacilos positivos para la tinción de Warthin Starry (Figura 2). Se realizó serología IgG $B$. henselae (IFI) que resultó positiva en título mayor a 1: 1.024, diagnosticándose como una infección

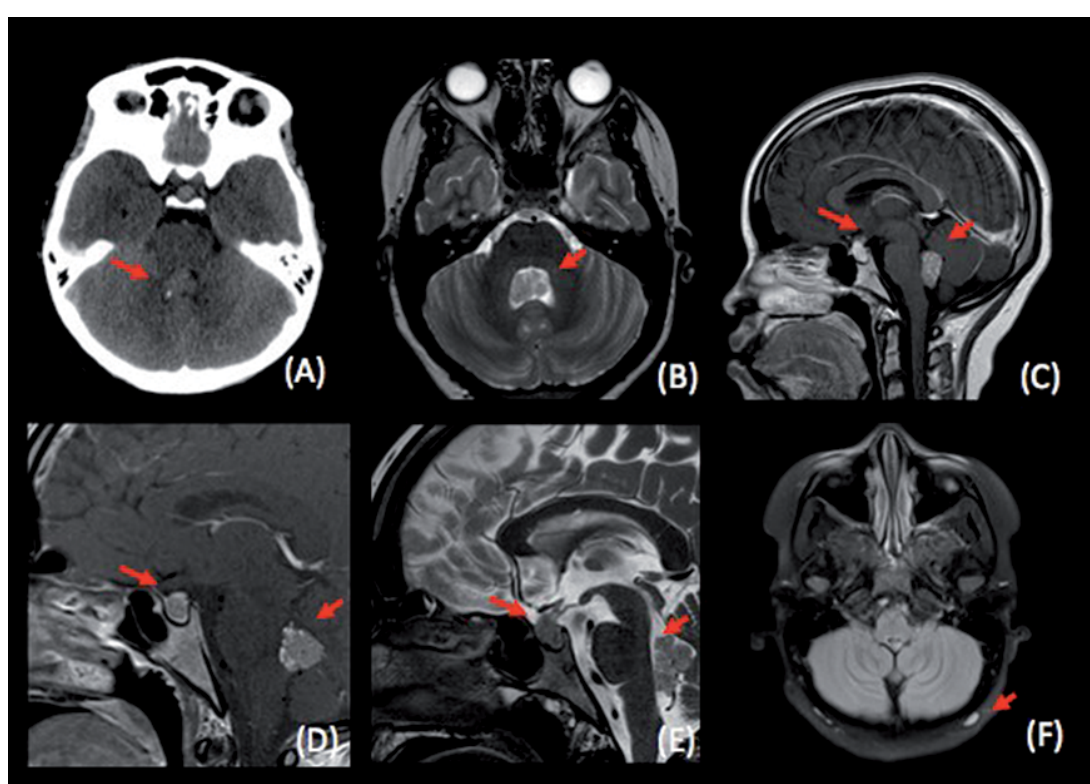

Figura 1. (A) TC que muestra proceso expansivo centrado en el cuarto ventrículo con una pequeña calcificación puntiforme. (B) y (E) RM de encéfalo y silla turca en secuencias T2-WI axial y sagital respectivamente, muestran proceso expansivo de alta señal centrado en el cuarto ventrículo. T1Gd-WI sagital de encéfalo y silla turca (C) y (D) respectivamente, muestran realce homogéneo del proceso expansivo intraventricular y discreto engrosamiento e impregnación del tallo hipofisiario y de la hipófisis. (E) Aumento de volumen hipofisiario en estudio dirigido a silla turca. (F) T2-FLAIR axial evidencia lesión nodular cervical posterior, sugerente de adenopatía.

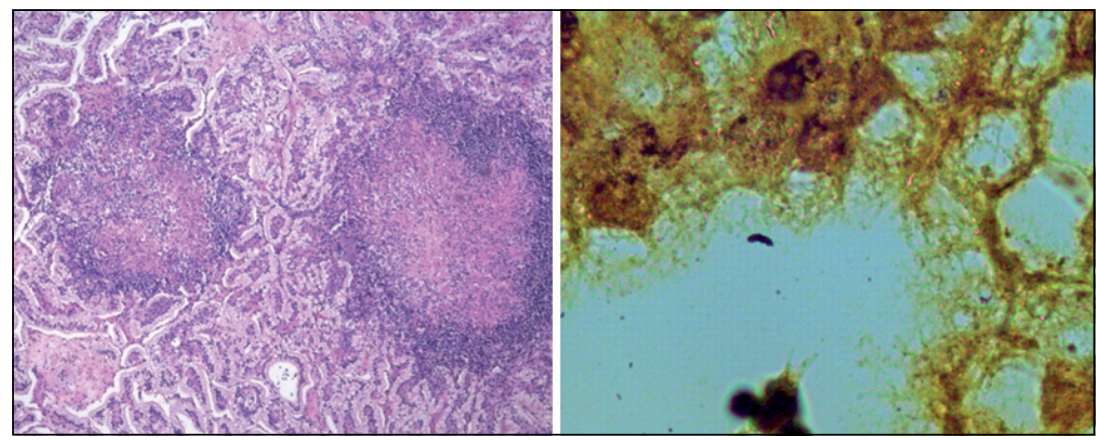

Figura 2. Imágenes de la biopsia del tumor tipo papiloma de plexo coroídeo. A. Tinción hematoxilinaeosina que muestra un proceso inflamatorio granulomatoso crónico. B. Tinción Warthin Starry positiva que evidencia la presencia de un bacilo. por $B$. henselae con compromiso del SNC. Se estudió la muestra de tejido tumoral con biología molecular para $B$. henselae, la que resultó negativa. Se reinternó e inició antibioterapia con ceftriaxona iv $2 \mathrm{~g}$ fraccionado cada 12 h y rifampicina $300 \mathrm{mg}$, dos veces al día.

El estudio de diseminación no mostró lesiones focales en el hígado y bazo, el cintigrama óseo y el fondo de ojo fueron normales. Una prueba de ELISA para VIH resultó no reactiva y un estudio básico de inmunidad resultó normal (medición de inmunoglobulinas y complemento). En un control de RM de encéfalo un mes y medio después de la cirugía, se evidenciaron cambios post-quirúrgicos de la fosa posterior, sin evidencia de remanente o recidiva; la lesión del infundíbulo hipofisiario mostraba cambios regresivos. Después de cuatro semanas de tratamiento antibacteriano bi-asociado, se dio de alta con cotrimoxazol vía oral, para completar seis semanas de terapia antibacteriana.

La paciente actualmente mantiene en control. La serología se encuentra con títulos en descenso (1:512). Persiste en rehabilitación con kinesioterapia motora y con psicoterapia, ya que se mantiene con leve ataxia a un año del inicio de sus síntomas.

\section{Discusión}

Esta paciente fue referida a nuestra institución con el diagnóstico de infección por $B$. henselae con compromiso de SNC bien fundamentado: una biopsia con bacilos visibles en la tinción Warthin Starry y el antecedente de contacto con gatos, sumado a la presencia de una serología positiva para $B$. henselae en títulos altos. Destacaba en la presentación inicial las adenopatías occipitales, sin evidencia de arañazo en esa zona. Frente al hallazgo de un granuloma asociado a un tumor de fosa posterior surgieron varias interrogantes: la descripción de casos similares en la literatura médica; una presunta relación causa-efecto entre ambas patologías o si la pre-existencia de una lesión tumoral pudiera haber favorecido la posterior instalación de un proceso infeccioso contiguo. Definir el tiempo de duración de los antimicrobianos fue un desafío para el equipo tratante.

El reservorio natural más frecuente de $B$. henselae son los gatos y se transmite al ser humano a través de rasguños o mordidas, principalmente gatos con menos de 1-2 años de edad ${ }^{1}$. En nuestra paciente, no había signos físicos de arañazos ni mordeduras, aunque sí tenía el antecedente de jugar con gatos. La incidencia real de la enfermedad por arañazo de gato es desconocida; en E.U.A se estima en 9,3 por 100.000 habitantes por año ${ }^{1,4}$. En Chile, Ferrés y cols, estudiaron el reservorio animal de la bacteria, siendo la prevalencia de IgG anti $B$. henselae en gatos de $85,6 \%$. Es interesante destacar que de ellos, $41 \%$ tenían 
hemocultivos positivos al momento de ser estudiados ${ }^{5}$. En niños chilenos, se documentó una seropositividad de $13,3 \% \mathrm{y}$, en veterinarios y técnicos veterinarios, $10,3 \%$ en el año $2006^{6}$.

\section{Clínica}

La gravedad y extensión del compromiso con el que se presenta la enfermedad, así como la respuesta patológica del organismo, difieren según el estado de inmunidad del hospedero $^{3,7}$. La enfermedad por arañazo de gato es la manifestación más típica de la infección por $B$. henselae ${ }^{1}$. Consiste generalmente en una única adenopatía regional cercana a la inoculación, aunque en 10-20\% de los casos se afecta más de un grupo ganglionar, de evolución aguda o subaguda. La ubicación de la lesión se relaciona con el drenaje linfático de la zona mordida o arañada ${ }^{1,8}$. En hospederos inmunocompetentes, la adenopatía se presenta una a tres semanas tras el contacto con gatos, pudiendo no haber compromiso del estado general, o aparecer síntomas leves de anorexia o artralgias, y fiebre, en un tercio de los pacientes ${ }^{1}$. La piel en torno a la lesión no presenta signos inflamatorios, salvo leve eritema y aumento de calor local ${ }^{1}$. En el caso presentado, la paciente presentó como su primera manifestación clínica adenopatías occipitales, que a los tres meses ya no estaban presentes. Fue tratada inicialmente con azitromicina, pero es difícil precisar si las adenopatías desaparecieron como efecto del tratamiento o fue la evolución natural de la enfermedad ya que, en general tienden a resolverse espontáneamente, en aproximadamente dos meses, rara vez persistiendo por más de 6 a 12 meses ${ }^{1}$. Además, debemos tener presente que existen algunos pacientes que no presentan lesiones de piel al momento del diagnóstico como fue nuestro caso.

Las presentaciones atípicas $(5-20 \%)^{9}$ se describen generalmente en pacientes inmunocomprometidos, siendo la ocular la más frecuente ${ }^{1,10}$, aunque también se puede presentar con compromiso hepato-esplénico, del SNC, óseo, endocárdico, compromiso pleuro-pulmonar, renal, hematológico y cutáneo ${ }^{1}$. El síndrome febril prolongado o síndrome mononucleósico pueden darse, con o sin compromiso inmunitario. En nuestra paciente se realizó estudio para VIH y pruebas de inmunidad básica, que resultaron normales, no pudiendo demostrarse una inmunosupresión. Compromiso por B. henselae de SNC y su relación con patología tumoral

En las manifestaciones asociadas al compromiso de SNC, el inicio de los síntomas suele ser entre dos días y dos meses posteriores a la aparición de la adenopatía ${ }^{1}$. La expresión clínica es variada; cefalea, convulsiones, ataxia, hemiplejía parcial, afasia, pérdida auditiva, neuroretinitis, meningo-radiculopatía, mielitis transversa, letargia y coma ${ }^{1}$. En nuestra paciente, la ataxia apareció después de dos semanas de fiebre y de las adenopatías occipitales, lo que concuerda con la literatura médica consultada, si bien éste es un síntoma inhabitual y podría también explicarse por la ubicación del tumor en la fosa posterior. El análisis citoquímico del LCR puede ser normal o mostrar pleocitosis con predominio linfocítico ${ }^{1,10}$, lo que corresponde a lo encontrado en este caso. El estudio con imágenes con TC o RM cerebral también puede ser normal y la evolución suele ser autolimitada ${ }^{10,11}$. El deterioro cognitivo y la muerte están reportados como evoluciones extremadamente infrecuentes ${ }^{1}$. En nuestro caso se mezclaron ambas sintomatologías, las asociadas al granuloma de SNC y las asociadas a la lesión tumoral.

En esta búsqueda no encontramos relación causal entre la infección por $B$. henselae con tumores de plexo coroídeo y sólo está descrito como parte del diagnóstico diferencial. Existen otros tumores asociados a la infección por Bartonella spp. como adenopatías malignas o tumores vasoproliferativos $^{1,2}$.

La asociación entre infección por $B$. henselae y tumores es secundaria a proliferación vascular. Aunque los mecanismos no están claramente definidos, se postula que la inhibición de la apoptosis de las células endoteliales, estimulando la producción de factor de crecimiento vascular endotelial ${ }^{12}$ y una reprogramación angiogénica de las células infectadas del huésped, pueden actuar como mecanismos con efecto sinérgico entre si $^{13}$. También se describe que $B$. henselae se adhiere e invade células hematopoyéticas humanas ${ }^{14}$. Ha sido propuesto que las células hematopoyéticas podrían transportar la bacteria a los tejidos periféricos, en particular, al endotelio de la microcirculación, donde iniciarían los desórdenes vasoproliferativos ${ }^{2}$. En animales como perros, lobos, caballos y gatos se han encontrado secuencias de ADN de $B$. henselae en tumores como hemangiopericitomas y angioendoteliomatosis ${ }^{15,16}$.

Las manifestaciones de tumores vasoproliferativos por $B$. henselae más significativas en humanos son la angiomatosis bacilar y peliosis bacilar. Estas presentaciones clínicas son propias de pacientes inmunocomprometidos, principalmente con infección por VIH, lo que sugiere que la supresión de mecanismos regulados por la inmunidad también juega un rol en la vasoproliferación mediada por $B$. henselae ${ }^{17}$. Los casos descritos en pacientes con angiomatosis bacilar inmunocompetentes, en realidad se han presentado en períodos en inmunosupresión transitoria como quimioterapia o tratamiento inmunosupresor posterior a trasplante ${ }^{2}$.

Los tumores del plexo coroídeo son neoplasias raras, entre ellos, se encuentra el papiloma del plexo coroídeo, un tumor papilar benigno que corresponde a $2-4 \%$ de los tumores de SNC en menores de 15 años y $20 \%$ en lactantes bajo un año ${ }^{18}$. La localización más frecuente en menores son los ventrículos laterales ${ }^{18}$. En adolescentes y adultos compromete el cuarto ventrículo y el ángulo cerebro-pontino $^{18}$. Histológicamente, se compone de 
tejido conectivo fibrovascular cubierto por una capa simple de células epiteliales con núcleos monomorfos ${ }^{19}$. Los tumores de plexo coroideo son bien vascularizados; tanto en papilomas como en carcinomas hay angiogénesis en distintos grados, entre otros, por el receptor del factor de crecimiento derivado de plaquetas (PDGFR) que se ha visto involucrado en la tumorigénesis. PDGFR es un receptor tirosina-kinasa con participación en el desarrollo del SNC, formación de vasos y hematopoyesis ${ }^{20}$. Se ha encontrado señalización aberrante en crecimiento de tumores, angiogénesis, reclutamiento estromal, invasión tumoral y metástasis ${ }^{20}$.

Por tanto, es difícil certificar si existió o no una relación causal entre el tumor y la patología infecciosa que presentó la paciente. Como se describe, existen mecanismos de angiogénesis y tumorigénesis que podrían estar relacionados entre el papiloma y la infección por Bartonella.

\section{Diagnóstico}

Las técnicas serológicas son un método indirecto de diagnóstico, pero es lo más utilizado hoy en día. La detección de IgG mediante inmunofluorescencia (IFI), presenta una sensibilidad de $88-100 \%$ y una especificidad de $92-98 \%{ }^{21}$, siendo considerada el estándar de oro; dado que en nuestro país, la infección y la enfermedad son endémicas, el valor de corte recomendado para asignar un valor diagnóstico de enfermedad actual es de 1:256 22 . Están descritos casos en los que los títulos de IgG disminuyen lentamente tras la infección y pueden permanecer positivos por más de dos años; sólo un cuarto de los pacientes se hacen seronegativos al cabo de un año $0^{10,11,23}$. En pacientes en que la serología resulte negativa y se tiene la fuerte sospecha clínica, se sugiere repetir el examen al cabo de dos a cuatro semanas y realizar una curva serológica, considerando un ascenso de al menos cuatro diluciones como significativo ${ }^{23}$. En nuestra paciente se repitió la serología al mes de evolución y se mantuvo alta, sin variación en el título, siendo esto esperable ya que los títulos pueden demorar años en negativizarse. Cumpliendo un año posterior al tratamiento, bajó sólo en una dilución.

Las opciones diagnósticas de la infección por $B$. henselae incluyen, dentro de los métodos directos, el cultivo, que es difícil y engorroso de realizar ${ }^{2,4}$. Otros métodos directos son, la visualización de granulomas en el estudio histológico, con formas bacterianas sugerentes de la etiología, en su interior, a través de la tinción de Warthin Starry, estudio inmunohistoquímico y la biología molecular ${ }^{1}$. En nuestra paciente el diagnóstico se realizó principalmente por el estudio histológico del tumor, en que como hallazgo se encontraron bacilos positivos para la tinción de Warthin Starry, corroborándose con la serología positiva para $B$. henselae en títulos muy elevados.

El estudio histológico de las lesiones revela un granuloma con múltiples microabscesos ${ }^{1}$. En la citología es frecuente observar linfocitos B monocitoides, histiocitos epitelioides, neutrófilos (en etapas más tardías) y necrosis supurativa $^{24}$. Ante el hallazgo de lesiones granulomatosas en la histología, se debe considerar como diagnóstico diferencial tuberculosis, linfoma, sarcoidosis, brucelosis, linfogranuloma venéreo, tularemia o esporotricosis ${ }^{24}$.

\section{Imagenología}

Dado al compromiso sistémico de la infección por $B$. henselae es posible encontrar múltiples hallazgos imagenológicos. Las adenopatías son frecuentes y detectables en ultrasonografía (US), TC y RM; en las cuales se evidencia un aumento de volumen de los linfonodos. La US demuestra adenopatías hiperémicas, con o sin necrosis central ${ }^{25}$. En un estudio ecográfico sobre 24 pacientes con enfermedad por arañazo de gato confirmada se identificó en $80 \%$ de ellos un tejido hipoecogénico con márgenes mínimamente irregulares y en $100 \%$ una hipervascularidad al Doppler del hilio central ${ }^{26}$, lo que es concordante con la capacidad de generar proliferación vascular que tiene Bartonella spp $^{2}$. La TC pesquisa lesiones lobuladas hipoatenuadas, con o sin un centro necrótico. En RM demuestran una señal intermedia en T1-WI, alta señal en T2-WI y realce periférico en T1Gd-WI ${ }^{27}$. El compromiso neurológico es poco frecuente e inespecífico, e incluye la meningoencefalitis, con sus hallazgos clásicos caracterizado por lesiones con incremento de señal en T2-WI encefálicas e incremento de señal en los surcos; y realce leptomeníngeo focal o difuso con el medio de contraste. Otros hallazgos descritos en RM son lesiones post convulsivas o post status epiléptico ${ }^{11,28}$, cuadro de encefalopatía posterior reversible, neuritis craneal y lesiones desmielinizantes ${ }^{25,29}$. La neuroretinitis se caracteriza por un engrosamiento de baja señal en T2 y de alta señal en FLAIR del aspecto posterior del globo ocular y/o realce posterior del globo ocular (retina y coroides), mácula o nervio óptico proximal ${ }^{25}$.

\section{Tratamiento}

El tratamiento etiológico de la infección por $B$. henselae es motivo de controversia, debido a que es un microrganismo intracelular y está ampliamente distribuido en eritrocitos y células endoteliales. Las bacterias del género Bartonella son susceptibles in vitro a varios antimicrobianos incluidos penicilinas, cefalosporinas, aminoglucósidos, cloranfenicol, tetraciclinas, macrólidos, rifampicina, fluoroquinolonas y cotrimoxazol. Existe discordancia entre la sensibilidad in vitro y la utilidad clínica de los diferentes antimicrobianos, posiblemente explicada por la ubicación intracelular de la bacteria ${ }^{1,3,7,23,30}$. Frente a las infecciones graves se recomienda utilizar dos antimicrobianos con diferentes mecanismos de acción para lograr una mejor erradicación ${ }^{7}$, fue por esto que nuestra paciente se trató con ceftriaxona y rifampicina ${ }^{31}$. 
El tratamiento con azitromicina por plazo de 5 días favorece la disminución del volumen de la adenopatía, lo que ha sido estimado en $20 \%$ en los primeros días, pero sin diferencias significativas a 30 días $^{3,7,30,32}$. Se ha observado beneficio clínico en el tratamiento de bartonelosis ocular y con compromiso de SNC utilizando, en combinación, rifampicina y doxiciclina. Aunque la duración del tratamiento no está definida, se prefiere esta asociación de antimicrobianos por la excelente penetración de doxiciclina en el $\mathrm{SNC}^{3,7,10,11,23,33,34}$.

Se recomienda tratamiento antimicrobiano prolongado, por al menos tres meses, en todos los pacientes inmunocomprometidos que cursen con infección por Bartonella spp., acompañando a la búsqueda de diseminación hematógena; los pacientes que presenten recaída, debieran utilizar profilaxis secundaria ${ }^{7,35,36}$.

No hay evidencia hasta el momento que avale el uso de corticoesteroides en las infecciones por Bartonella spp., aunque se han utilizado para el manejo de abscesos hepato-esplénicos considerados como cambios post inflamatorios ${ }^{1,23,24}$.

En suma, la decisión y elección del tratamiento de las infecciones causadas por Bartonella spp. se basa en la experiencia personal del equipo tratante, la opinión de expertos y el conocimiento de la susceptibilidad antimicrobiana in vitro descrita ${ }^{30}$. Fue por estos motivos y además por la disponibilidad del fármaco, que en nuestra paciente se decidió continuar tratamiento ambulatorio con cotrimoxazol hasta completar seis semanas. Actualmente no hay evidencia de recaída o recidiva tanto clínica como en imágenes.

Agradecimientos: A Alejandra Henríquez por facilitar imágenes de anatomía patológica.

\section{Resumen}

La infección por Bartonella henselae es una enfermedad prevalente en nuestro país. En general, se presenta como la enfermedad por arañazo de gato o un síndrome febril prolongado. Existen manifestaciones atípicas dentro de las cuales está el compromiso óseo, cardíaco, hepatoesplénico y del sistema nervioso central. Se presenta el caso de una adolescente con historia de vómitos, fiebre y ataxia, en que se diagnosticó una infección por Bartonella henselae con compromiso del sistema nervioso central, asociada a un papiloma del plexo coroídeo. Este caso corresponde a una presentación inusual, de difícil diagnóstico. Su tratamiento es motivo de controversia, tanto en si es necesario tratar, la elección del antimicrobiano, como su duración.

\section{Referencias bibliográficas}

1.- Massei F, Gori L, Macchia P, Maggiore G. The expanded spectrum of bartonellosis in children. Infect Dis Clin North Am 2005; 19: 691-711.

2.- Pulliainen AT, Dehio C. Persistence of Bartonella spp. stealth pathogens: from subclinical infections to vasoproliferative tumor formation. FEMS Microbiol Rev 2012; 36: 563-99.

3.- Angelakis E, Raoult D. Pathogenicity and treatment of Bartonella infections. Int J Antimicrob Agents 2014; 44: 16-25.

4.- $\quad$ Schutze G, Jacobs R. Chapter 160: Bartonella Species (Cat-Scratch Disease). Principles and Practice of Pediatric Infectious Diseases. Long S, Pickering L, Prober C, Adderson E, Daum R, Brook I, et al. 4th edition, 2012, Elsevier, Edinburgh, p. 856-61.

5.- Ferrés G M, Abarca V K, Godoy M P, García C P, Palavecino R E, Méndez R G, et al. Presencia de Bartonella henselae en gatos: cuantificación del reservorio natural y riesgo de exposición humana de esta zoonosis en Chile. Rev Med Chile 2005; 133: 1465-71.

6.- Ferrés G M, Abarca V K, Prado D P, Montecinos P L, Navarrete C M, Vial P. Prevalencia de anticuerpos contra Bartonella henselae en niños, en adolescentes y en una población de riesgo ocupacional en Chile. Rev Med Chile 2006; 134: 863-7.

7.- Rolain J M, Brouqui P, Koehler J E, Maguina C, Dolan M J, Raoult D. Recommendations for treatment of human infections caused by Bartonella species. Antimicrob Agents Chemother 2004; 48: 1921-33.

8.- Carithers H A. Cat-scratch disease. An overview based on a study of 1,200 patients. Am J Dis Child 1985; 139: 1124-33.

9.- García J C, Núñez M J, Castro B, Fernández J M, López A, Portillo A, et al. Hepatosplenic cat scratch disease in immunocompetent adults: report of 3 cases and review of the literature. Medicine 2014; 93: 267-79.

10.- Pinto Jr V L, Curi A L, Pinto Ada S, Nunes E P, Teixeira M de L, Rozental T, et al. Cat scratch disease complicated with aseptic meningitis and neuroretinitis. Braz J Infect Dis 2008; 12: 158-60.

11.- Wyllie E, Rincon S P, Pierce V M. Case records of the Massachusetts General Hospital. Case 16-2015. A 9-year-old girl with loss of consciousness and seizures. N Engl J Med 2015; 372: 2050-8.

12.- Dehio C. Recent progress in understanding Bartonella-induced vascular proliferation. Curr Opin Microbiol 2003; 6: 61-5.
13.- Kaiser P O, Riess T, O'Rourke F, Linke D, Kempf V A. Bartonella spp.: throwing light on uncommon human infections. Int J Med Microbiol 2011; 301: 7-15.

14.- Salvatore P, Casamassimi A, Sommese L, Fiorito C, Ciccodicola A, Rossiello R, et al. Detrimental effects of Bartonella henselae are counteracted by $\mathrm{L}$-arginine and nitric oxide in human endothelial progenitor cells. Proc Natl Acad Sci U S A 2008; 105: 9427-32.

15.- Beerlage C, Varanat M, Linder K, Maggi R G, Cooley J, Kempf V A, et al. Bartonella vinsonii subsp. berkhoffii and Bartonella henselae as potential causes of proliferative vascular diseases in animals. Med Microbiol Immunol 2012; 201: 319-26.

16.- Varanat M, Travis A, Lee W, Maggi R G, Bissett S A, Linder K E, et al. Recurrent osteomyelitis in a cat due to infection with Bartonella vinsonii subsp. berkhoffii genotype II. J Vet Intern Med 2009; 23 : 1273-7.

17.- Koehler J E, Sánchez M A, Garrido C S, Whitfeld M J, Chen F M, Berger T G, et al. Molecular epidemiology of Bartonella infections in patients with bacillary angiomatosis-peliosis. N Engl J Med 1997; 337: 1876-83.

18.- Paulus W, Brandner S, Hawkins C, Tihan T. 
Choroid plexus tumor. Louis D N, Ohgaki K, Wiestler O D, Cavenee W K, Ellison D W, Perry A, et al. WHO Classification of tumours of the central nervous system. 4th edition, Lyon: International Agency for Research on Cancer (IARC), 2016, p.123-30.

19.- Rickert C H, Paulus W. Tumors of the choroid plexus. Microsc Res Tech 2001; 52: 104-11.

20.- Safaee M, Oh M C, Bloch O, Sun MZ, Kaur G, Auguste K I, et al. Choroid plexus papillomas: advances in molecular biology and understanding of tumorigenesis. Neuro Oncol 2013; 15: 255-67.

21.- Regnery R L, Olson J G, Perkins B A, Bibb W. Serological response to "Rochalimaea henselae" antigen in suspected cat-scratch disease. Lancet 1992; 339: 1443-5.

22.- Abarca K, Winter M, Marsac D, Palma C, Contreras A M, Ferrés M. Exactitud y utilidad diagnóstica de la IgM en infecciones por Bartonella henselae. Rev Chilena Infectol 2013; 30: 125-8.

23.- Täger F M, Jahnsen K J, Mediavilla R M, Burgos L R. Bartonelosis ocular: Reporte de tres casos. Rev Chilena Infectol 2008; 25: 5863.

24.- Choi A H, Bolaris M, Nguyen D K, Panosyan E H, Lasky J L 3rd, Duane G B. Clinicocytopathologic correlation in an atypical presentation of lymphadenopathy with review of literature. Am J Clin Pathol 2015;
143: 749-54.

25.- Rohr A, Saettele M R, Patel S A, Lawrence C A, Lowe L H. Spectrum of radiological manifestations of paediatric cat-scratch disease. Pediatr Radiol 2012; 42: 1380-4.

26.- Bernard S A, Walker E A, Carroll J F, KlassenFischer M, Murphey M D. Epitrochlear cat scratch disease: unique imaging features allowing differentiation from other soft tissue masses of the medial arm. Skeletal Radiol 2016; 45: 1227-34.

27.- Wang C W, Chang W C, Chao T K, Liu C $\mathrm{C}$, Huang G S. Computed tomography and magnetic resonance imaging of cat-scratch disease: a report of two cases. Clin Imaging 2009; 33: 318-21.

28.- Ogura K, Hara Y, Tsukahara H, Maeda M, Tsukahara M, Mayumi M. MR signal changes in a child with cat scratch disease encephalopathy and status epilepticus. Eur Neurol 2004; 51: 109-10.

29.- Brinar V V, Habek M. Rare infections mimicking MS. Clin Neurol Neurosurg 2010; 112: 625-8

30.- Prutsky G, Domecq J P, Mori L, Bebko S, Matzumura M, Sabouni A, et al. Treatment outcomes of human bartonellosis: a systematic review and meta-analysis. Int J Infect Dis 2013; 17: e811-9.

31.- Antimicrobial Therapy According to Clinical Syndromes. Nelson's Pediatric Antimicrobial Therapy. Bradley J, Nelson J, Barnett E, Cantey
J, Kimberlin D, et al., 22nd edition, 2016, American Academy of Pediatrics, Elk Grove Village, p. 98.

32.- Bass J W, Freitas B C, Freitas A D, Sisler C L, Chan D S, Vincent J M, et al. Prospective randomized double blind placebo-controlled evaluation of azithromycin for treatment of cat-scratch disease. Pediatr Infect Dis J 1998; 17: 447-52.

33.- Reed J B, Scales D K, Wong M T, Lattuada C P Jr, Dolan M J, Schwab I R. Bartonella henselae neuroretinitis in cat scratch disease. Diagnosis, management, and sequelae. Ophthalmology 1998; 105: 459-66.

34.- Smith R A, Scott B, Beverley D W, Lyon F, Taylor R. Encephalopathy with retinitis due to cat-scratch disease. Dev Med Child Neurol 2007; 49: 931-4.

35.- Bonatti H, Méndez J, Guerrero I, Krishna M, Ananda-Michel J, Yao J, et al. Disseminated Bartonella infection following liver transplantation. Transpl Int 2006; 19: 683-7.

36.- Kaplan J E, Benson C, Holmes K K, Brooks J T, Pau A, Masur H, et al. Guidelines for prevention and treatment of opportunistic infections in HIV-infected adults and adolescents: recommendations from $\mathrm{CDC}$, the National Institutes of Health, and the HIV Medicine Association of the Infectious Diseases Society of America. MMWR Recomm Rep 2009; 58 (RR-4): 1-207. 